Pacific Journal of Mathematics

OPERATIONAL CALCULUS OF LINEAR RELATIONS 


\section{OPERATIONAL CALCULUS OF LINEAR RELATIONS}

\section{RICHARD ARENS}

1. Introduction. Let $X$ and $Y$ be linear spaces, and $T$ a linear subspace of $X \oplus Y$. We call $T$ a linear relation to indicate our interest in those constructions with $T$ which generalize those carried out when $T$ is single-valued [4].

Properly many-valued linear relations arise naturally from operators $T$ when $T^{-1}$ or $T^{*}$ is contemplated in cases where they are not singlevalued. One advantage of not dismissing $T^{*}$ when it is not singlevalued is that $T^{* *}=T$ if and only if $T$ is closed (for the details, see 3.34, below.) A more superficial attraction is that linear relations, even self-adjoint linear relations in Hilbert space can exhibit phenomena (unbounded spectrum, domain $\neq X$ ) in finite-dimensional spaces which linear operators exhibit only in infinite-dimensional spaces.

We present an outline of the paper. In $\S 2$ we define $p(T)$ where $p$ is a polynomial with coefficients in the field $\Phi$ involved in $X$. We prove that $(p q)(T)=p(T) q(T),(p \circ q)(T)=p(q(T))$, and point out that sometimes $(p+q)(T) \neq p(T)+q(T)$, etc.

In $\S 3$ we turn to relations in dual pairs. In this situation, adjoints can be defined. We build an automorphism $\lambda \rightarrow \bar{\lambda}$ of $\Phi$ into the theory of dual pairs, so as not to exclude the Hilbert space situation, which dual pairs are intended to imitate. (Thus the transpose is a special kind of adjoint.) Closedness is defined algebraically, but in a way compatible with the topological concept. Closure of $T^{*}$ and other algebraic properties of ${ }^{*}$ are established. Finally, it is shown that if $T$ is closed and its resolvent is not void then $p(T)$ is also closed.

Section 4 considers the self-dual case. We give a simple condition (4.3) always true in Hilbert space, that $T^{*} T$ be self-adjoint, $T$ being closed. In $\S 5$ we give the spectral analysis of self-adjoint linear relations in Hilbert space. In a 1:1 manner these correspond to the unitary operators, via the Cayley transform. However, it can be shown directly that $X$ is the direct sum of orthogonal subspaces $Y, Z$ which reduce $T\left(=T^{*}\right)$ giving in $Z$ a self-adjoint operator and in $Y$ the inverse of the zero-operator.

2. Linear relations. A relation $T$ between members of a set $X$ and members of a set $Y$ is merely a subset of $X \times Y$. For $x \in X, T(x)=$ $\{y:(x, y) \in T\}$. The domain of $T$ consists of those $x$ such that $T(x)$ is not void. $T$ is called single-valued if $T(x)$ never contains more than one element. The range of $T$ is the union of all $T(x)$.

Received April 13, 1960. 
If $T$ is as above and $S \subset Y \times Z$, then $S \circ T=\{(x, z):(x, y) \in T$, $(y, z) \in S$ for some $y\}$. We shall write this $S T$. Finally, $T^{-1}=$ $\{(y, x):(x, y) \in T\}$. The range of $T$ is the domain of $T^{-1}$.

If $X$ and $Y$ are linear spaces over a field $\Phi$ then $X \oplus Y$ is $X \times Y$ with the usual linear structure. A linear relation $T$ between members of $X$ and members of $Y$ is a linear subspace of $X \oplus Y$. Linearity is characterized by

2.01

$$
\alpha T\left(x_{1}\right)+\beta T\left(x_{2}\right) \subset T\left(\alpha x_{1}+\beta x_{2}\right), \quad\left(\alpha, \beta \in \Phi ; x_{1}, x_{2} \in X\right) .
$$

The null space of $T$ is the class of $x$ such that $(x, 0) \in T$. It is easy to see that

2.02 if $S$ and $T$ are linear relations with the same null space, and the same range, then $S \subset T$ only if $S=T$.

Let $L$ be a linear subspace of $X$, and $\lambda$ an element of $\Phi$. Then $\lambda_{L}$ denotes the single valued operator defined on $L$ by $\lambda_{L}=\{(x, \lambda x): x \in L\}$. The unit of $\Phi$ we denote by 1 . Thus $1_{L}$ has a meaning according to the preceeding agreement. For $T$ a linear relation with range $L$, we define $\lambda T$ as $\lambda_{L} T$. The zero of $\Phi$ we denote by 0 . Thus $O T$ is not $O_{x}$, but $O_{L}$ where $L$ is the domain of $T$.

Addition of linear relations $S, T$ is defined as follows:

$S+T=\{(x, y): y=s+t$ for some $s, t$ such that $(x, s) \in S,(x, t) \in T\}$.

The linear relations in $X \oplus X$ do not form a linear space, let alone a linear algebra. We list algebraic properties partly for use later, but mainly to call attention, as it were, to those that are lacking.

2.1 THEOREM. The operations ' $\circ$ ' and $(+)$ are associative, ' + ' $i s$ commutative. Let $R, S, T$ be linear relations. Then

2.11 domain of $R=X \Leftrightarrow 1_{X} \subset R^{-1} R$;

$2.12 R$ is single-valued $\Leftrightarrow R R^{-1} \subset 1_{L}, L=$ range of $R$;

$2.13 \lambda \in \Phi \Rightarrow \lambda(S T)=(\lambda S) T=S(\lambda T)=S T \lambda_{L}, L=$ domaiu of $T$;

$2.14 R \subset S \Rightarrow R+T \subset S+T, R T \subset S T, T R \subset T S, R^{-1} \subset S^{-1}$;

$2.15 R S+R T \subset R(S+T)$, with equality when the domain of $R$ coincides with the whole space;

$2.16(S+T) R \subset S R+T R$, with equality when $R$ is single-valued;

$2.17(S T)^{-1}=T^{-1} S^{-1}$.

The proof of these may be left to the reader.

We say $S$ and $T$ commute is $S T=T S$. Suppose $S R=R S, T R=R T$. Then $(S+T) R \subset R(S+T)$. The equality may not hold, as the example $S=-T=1_{X}$, domain of $R \neq X$, will show.

$T^{n}$ is defined as $T^{n-1} T$, as usual. If $T^{n}$ appears in a formula where $n=0$ is allowed, then $T^{0}$ stands for $1_{x}$.

These things can all be extended to the case of moduls over a ring $\Phi$. However, we now turn to a lemma whose proof requires that $\Phi$ be a 
field.

For the remainder of $\S 2, T$ will denote a linear relation in $X \oplus X$, and for $\lambda \in \Phi$, we write just ' $\lambda$ ' for ' $\lambda_{x}$ '.

It is clear that $\alpha_{0}+\alpha_{1} T+\cdots+\alpha_{n} T^{n}$ has for its domain, just the domain of $T^{n}$. This is true even if $\alpha_{n}=0$ ! If a polynomial $p$ has coefficients $\alpha_{0}, \alpha_{1}, \cdots, \alpha_{n}$, then by $p(T)$ we mean $\alpha_{0}+\alpha_{1}+\cdots+\alpha_{n} T^{n}$ provied $\alpha_{n} \neq 0$. Otherwise we omit $\alpha_{n}$ and consider whether $\alpha_{n-1} \neq 0$, etc. If $\alpha_{n} \neq 0$ and $\alpha_{i}=0$ for some $i<n$, then it does not matter whether $\alpha_{i}$ is omitted or not (but we have already agreed to retain it) because, for example $T^{3}+0 T=T^{3}$.

The next lemma settles a little difficulty that arises in the 'multiplevalued' situation. It enables us to include the multiple valued case in the succeeding theorem, whose substance is that the usual laws of algebra apply to the multiplication of linear polynomials in $T$. The importance of this theorem is based on the natural fear that even in the single valued case (see $2.15,2.16$ ), factoring might produce a proper extension of the "multiplied-out" polynomial.

2.2 Lemma. Let $(x, y) \in \alpha_{0}+\alpha_{1} T+\cdots+\alpha_{n} T^{n}$, where $\alpha_{n} \neq 0$. Then there exist $y_{0}, y_{1}, \cdots, y_{n}$ such that

$$
y_{0}=x, \sum_{i=0}^{n} \alpha_{i} y_{i}=y
$$

and

2.22

$$
\left(y_{i-1}, y_{i}\right) \in T
$$$$
(i=1, \cdots, n) \text {. }
$$

Proof. Assume that for some $j$, we have $y_{0}, y_{1}, \cdots, y_{n}$ such that 2.21 holds, and (instead of 2.22)

$$
\left(y_{i-1}, y_{i}\right) \in T
$$

and

$$
\left(x, y_{i}\right) \in T^{i}
$$$$
(1 \leqq i \leqq n)
$$

Let $k$ be the next integer greater than $j$ such that $\alpha_{k} \neq 0$. We shall establish $(k)$. This will suffice to prove the lemma.

Because $\alpha_{k} \neq 0$ we can find $\lambda_{1}, \cdots, \lambda_{j}$ such that, for $1 \leqq h \leqq j$,

$$
\sum_{m=k-j+h}^{k} \alpha_{m} \lambda_{j-k+m+1-h}=\alpha_{h} .
$$

We can find $z_{1}, z_{2}, \cdots, z_{k}$ where $z_{k}=y_{k}$ and $\left(x, z_{1}\right),\left(z_{1}, z_{2}\right), \cdots,\left(z_{k-1}, z_{k}\right) \in T$. This implies that $\left(0, y_{1}-z_{1}\right) \in T$, and $\left(y_{i-1}-z_{i-1}, y_{i}-z_{i}\right) \in T$ for $i \leqq j$. Now we define $w_{0}, w_{1}, \cdots, w_{n}$ as follows. $w_{0}=x, w_{1}=z_{1}$, for $1 \leqq m \leqq k$,

$$
w_{m}=z_{m}+\sum_{i=1}^{j-k+m} \lambda_{i}\left(y_{j-k+m+1-i}-z_{j-k+m+1-i}\right)
$$


while $w_{k+1}=y_{k+1}, \cdots, w_{n}=y_{n}$. It is clear that $\left(w_{i-1}, w_{i}\right) \in T$ for $i \leqq k$, and $\left(x, w_{i}\right) \in T^{i}$ for all $i$. There remains only the question, does $\sum \alpha_{i} w_{i}=y$, or, equivalently, does

$$
\sum_{m=1}^{k} \alpha_{m}\left(w_{m}-y_{m}\right)=0 ?
$$

The sum in 2.24 has the value

$$
\sum_{m=1}^{k-1} \alpha_{m}\left(z_{m}-y_{m}\right)+\sum_{m=1}^{k} \sum_{i=1}^{j-k+m} \alpha_{m} \lambda_{i}\left(y_{j-k+m+1-i}-z_{j-k+m+1+i}\right) .
$$

It is not hard to verify that for $0 \leqq h<k$ the coefficient of $y_{h}-z_{h}$ in this sum is

$$
-\alpha_{h}+\sum_{m=k-j+h}^{k} \alpha_{m} \lambda_{j-k+m+1-h},
$$

where the $\sum$-term is understood to be absent when $k-j+h>k$. These $\lambda$ were chosen in order to make this vanish for $0 \leqq h \leqq j$. For $j<h<k, \alpha_{h}=0$; since $k<k-j+h$, the $\sum$ term is absent. Thus the sum in 2.24 is 0 , and this concludes the proof of the Lemma (2.2).

N.B. This lemma does not imply that $T$ could be cut down to a linear operator $U$ whose domain contains $c, U x, \cdots$, and $U^{n-1} x$, where

$$
\sum_{m=0}^{n} \alpha_{m} U^{m}(x)=y,
$$

for $x$ could be 0 and $y$ be not 0 .

2.3 THEOREM. Let $p$ and $q$ be two polynomials with coefficients in $\Phi$. Then

2.31

$$
(q p)(T)=q(T) p(T) .
$$

Proof. Suppose the degrees of $p$ and $q$ are $m$ and $n$ respecively. Let $p(\xi)=\alpha_{0}+\alpha_{1} \xi+\cdots+\alpha_{m} \xi^{m}$. Mutatis mutandis, let the coefficients of $q$ and $q p$ be $\beta_{j}$ and $\gamma_{k}$.

Now suppose $(x, y) \in(p q)(T)$. By 2.2 there exist $x_{1}, \cdots, x_{m+n}$ such that $\left(x_{k-1}, x_{k}\right) \in T$ for $k=1, \cdots, m+n$ where $x_{0}=x$, and $\sum \gamma_{k} x_{k}=y$. Let $y_{j}=\sum_{i=1}^{m} d_{i} x_{i+j}$ for $j=0, \cdots, n$. Then $\left(x, y_{0}\right) \in p(T)$ and $\left(y_{j-1}, y_{j}\right) \in T$. Let $z=\sum_{j=0}^{n} \beta_{j} y_{j}$, so that $\left(y_{0}, z\right) \in q(T)$. Then $(x, z) \in q(T) p(T)$. But obviously $z=\sum \gamma_{k} x_{k}=y$. This shows that $(q p)(T) \subset q(T) p(T)$.

Now suppose $(x, z) \in q(T) p(T)$. Then there must exist $y$ such that $(x, y) \in p(T)$ and $(y, z) \in q(T)$. By 2.2 we can find $x_{0}, \cdots, x_{m}$ and $y_{0}, \cdots, y_{n}$ (where $x_{0}=x$, and $y_{0}=y$ ) such that $\sum \alpha_{i} x_{i}=y$ and $\sum \beta_{j} y_{j}=z$. We now turn to the free linear space $\Xi$ (over $\Phi$ ) generated by elements $\xi_{0}, \cdots, \xi_{m}, \eta_{1}, \cdots, \eta_{n}$. In $\Xi$ we define a linear operator $S$, whose domain is spanned by $\xi_{0}, \cdots, \eta_{n-1}$, as follows:

$S\left(\xi_{i-1}\right)=\xi_{i}(i=1, \cdots, m), S\left(\eta_{0}\right)=\eta_{1}$, where $\eta_{0}=\sum \alpha_{i} \xi_{i}$, and $S\left(\eta_{j}\right)=\eta_{j+1}$ 
$(j=1, \cdots, n-1)$. We can map $\Xi$ linearly into $X$ by a mapping $f$ which sends $\xi_{i}$ into $X_{i}$, and $\eta_{\jmath}$ into $y_{\jmath}$. This mapping has the property that for $\xi$ in the domain of $S,(f(\xi), f(S \xi)) \in T$. Derivable from this is that if $r$ is a polynominal and $r(S) \xi$ is defined some $\xi$ in $\Xi$ then $(f(\xi)$, $f(r(S) \xi)) \in r(T)$. We apply this to $\xi=\xi_{0}$ and $r=q p$. It is easy to see that $p(S)\left(\xi_{0}\right)=\eta_{0}$, whence $f(q p(S))\left(\xi_{0}\right)=f\left(\sum \beta_{j} \eta_{j}\right)=\sum \beta_{j} y_{j}=z$, and $(x, z) \in(q p)(T)$.

This completes the proof of 2.3 .

[Further remarks on polynomials of relations. Inspection of the first argument in the proof of 2.3 yields the following result.

2.32 Theorem. Let $p$ and $q$ be as in 2.3. Then

2.33

$$
(p+q)(T) \subset p(T)+q(T) .
$$

The '=' does not always hold. While

2.34

$$
\left(\sum \alpha_{i}\right) T=\Sigma\left(\alpha_{i} T\right)
$$

hold when $\sum \alpha_{i} \neq 0$, it does not hold when $\sum \alpha_{i}=0$, some $\alpha_{i} \neq 0$, and $T$ is not single-valued.

As the assertion connected with 2.34 implies, the reason that 2.33 cannot be strengthened to an inequality, is that $T-T$ is not 0 times some relation, if $T$ is not single-valued. We close this little discourse on the peculiarities of many-valued relations by showing that the difficulty arises only with the terms of highest order.

2.35 Theorem. Let $p, q$ be as above, and suppose the sum of their leading coefficients is not 0 . Then $(p+q)(T)=p(T)+q(T)$.

Proof. We combine the monomials of like degree on the right, and use 2.34 in each case. Eventually one may have to apply the following

\subsection{Lemma. If $n \geqq k$ then $T^{n}=T^{n}+\lambda\left(T^{k}-T\right)$.}

Proof. Let $(x, y)$ belong to the right side. Then $y=u+v$ where $(x, u) \in T^{n}+\lambda T^{k}$ and $(x, v) \varepsilon-\lambda T^{k}$. From 2.2 we obtain $u_{0}, \cdots, u_{n}$ which are successively T-related, $u_{0}=x, u_{n}+\lambda_{u_{k}}=u$. Therefore $\lambda_{u_{k}}+v \in T^{k}(0)$, whence $u_{n}+\lambda_{u_{k}}+v \in T^{k}\left(u_{n-k}\right) \subset T^{n}(x)$. Thus $(x, y) \in T^{n}$.

2.37 Theorem. Let $q$ and $p$ be polynomials. Then $(q \circ p)(T)=q(p(T))$.

Proof. The polynominal $q \circ p$ is the result of substituting $p$ into $q$, by definition. The leading coefficients may be taken as not zero. We can multiply out the terms $\beta_{j} p(T)^{j}$ on the right side, without affecting 
that sum, by 2.3. (The associative law holds for addition.) We can arrange the sum as a polynominal, by virtue of 2.35 there being in fact at all times a unique term $\alpha_{m} \beta_{n} T^{m+n}$ of highest degree. The resulting polynomial is of course $(q \circ p)(T)$, for formal reasons.]

We now make some definitions which coincide with the usual ones for closed operators in $F$-spaces. We call a linear relation $T$ resolvable if $T^{-1}$ is single-valued with domain $X$ (that is, by 2.11 , if $T^{-1} T \subset 1_{X} \subset T T^{-1}$. If $T^{-1} T=1=T T^{-1}$ we call $T$ regular.)

2.4 Proposition. The product of (finitely many pairwise) commuting linear relations is resolvable only if, and if, each factor is resolvable.

Proof. It is inevitable and sufficient to consider the case of two factors. If these are resolvable, so is their product. The criterion $T^{-1} T \subset 1 \subset T T^{-1}$ can be used here.

If on the other hand, a linear relation $S$ is not resolvable, then either $(x, 0) \in S$ for some $x \neq 0$, or the range $\neq X$. Accordingly, $T S$ or $S T$ shares the defect. (This sufficies for 2.4).

The resolvent set of a linear relation $T$ is the class of $\lambda$ in $\Phi$ for which $T-\lambda$ (by which we mean $T-\lambda 1_{x}$ ) is resolvable; and its complement is the spectrum $\sigma(T)$ of $T$.

2.5 (Spectral polynomial theorem). Let $\Phi$ be algebraically closed, and let $p$ be a polynomial over $\Phi$. Then $\sigma(p(T))=p(\sigma(T))$, where by the latter is meant the class of $p(\lambda), \lambda \in \sigma(T)$.

Proof. For $\mu \in \Phi$ we can write

$$
p(T)-\mu=\alpha\left(T-\lambda_{1}\right) \cdots\left(T-\lambda_{n}\right), \mu=p\left(\lambda_{1}\right)=\cdots=p\left(\lambda_{n}\right)
$$

where $T-\lambda_{1}, \cdots, T-\lambda_{n}$ commute.

If $\mu \in \sigma(p(T))$ then $p(T)-\mu$ is not resolvable, whence (by 2.4) some $\lambda_{i} \in \sigma(T)$, or $\left.\mu \in p(T)\right)$. If $\mu \in p(T)$ ) then $\mu=p(\lambda), \lambda \in \sigma(T)$, and so $\lambda=\lambda_{i}$ for some $i$. Then $p(T)-\mu$ has a non-resolvable factor, and so is not resolvable. Therefore $\mu \in \sigma(p(T))$. This proves 2.5.

We have defined the sum (and difference) of two linear subspaces $U$ and $V$ (say) of $X \oplus Y$, but occasionally one is concerned with the linear subspace of $X \oplus Y$ which they span. We will have to use some other symbol for this, and we choose

$$
U \neq V \text {. }
$$

Our purpose is to prove the following

2.61 THEOREM. The range of $1-V^{-1} U$ is the null-space of $U \neq V$, and the null-space of $1-V^{-1} U$ is the domain of $U \cap V$. 
Proof. Let $(x, z) \in 1-V^{-1} U$. Then $(x, z-x) \varepsilon-V^{-1} U$ whence $(x, y) \in U$ and $(y, x-z) \varepsilon-V^{-1}$, for some $y$. Therefore $(z-x,-y) \in V$ and so $(z, 0) \in U \neq V$. If moreover, $z=0$ (so that $x$ is in the nullspace) then $(-x,-y)$ and thus $(x, y)$ belongs to $V$ and thus $x \in \operatorname{dom} U \cap V$. The reverse inclusions can be established by reversing the steps of this argument.

3. Adjoints. For the formalism of adjoints, it is good to suppose that the field $\Phi$ has an involutory automorphism

$$
\lambda \rightarrow \bar{\lambda},
$$

and we shall do so. Whether $\Phi$ admits a non-trivial involution or not, one can base the discussion on the identity. Thus the discussion includes the transpose.

Let $X, A$ be two linear spaces over $\Phi$. We shall say $X, A$ are $a$ $(\Phi,-)$ dual pair (or, more briefly, a dual pair) is there is a non-degenerate bi-additive, $\Phi$-valued form $<$, $>$ defined on $X \oplus A$, linear in first argument, and semi-linear in the second:

$$
\langle x, \lambda x\rangle=\bar{\lambda}\langle x, a\rangle \text {. }
$$

Let $Y, B$ be another $(\Phi,-)$ dual pair. Let $T$ be a linear relation between elements of $X$ and elements of $Y$, i.e., let $T$ be a linear subspace of $X \oplus Y . \quad X \oplus Y, A \oplus B$ form a $(\Phi,-)$ dual pair, in a natural way:

$$
\langle(x, y),(a, b)\rangle=\langle x, a\rangle+\langle y, b\rangle .
$$

The adjoint $T^{*}$ is defined as follows:

3.11

$$
T^{*}=\{(b, a):\langle x, a\rangle=\langle y, b\rangle \text { for all }(x, y) \in T\} .
$$

$T^{*}$ is (evidently) a linear subspace of $B \oplus A$.

For a linear subspace $U$ of $B \oplus A$ we define

$$
U^{*}=\{(x, y):\langle x, a\rangle=\langle y, b\rangle \text { for all }(b, a) \in U\} .
$$

It is usually supposed that 3.12 need hardly be written down, once 3.11 is presented. We mention three obvious properties of this process (or, rather, these processes. See $\S 4$ )

$$
T \subset T^{* *}, S \subset T \Rightarrow T^{*} \subset S^{*}
$$

3.21

$$
(\lambda T)^{*}=\bar{\lambda} T^{*}
$$

$$
\left(T^{-1}\right)^{*}=\left(T^{*}\right)^{-1} \text {. }
$$

For a subset $M$ of $X$, let

$$
M^{\perp}=\{a:\langle x, a\rangle=0 \text { for all } x \in M\}
$$


while if $M \subset A$ then

3.24

$$
M^{\perp}=\{x:\langle x, a\rangle=0 \text { for all } a \in M\} .
$$

In this sense $(c f .[4])$

3.3

$$
T^{*}=\left(-T^{-1}\right)^{\perp} \text {. }
$$

In 3.3 we have in mind the natural pairing of $Y \oplus X$ and $B \oplus A$, of course.

Again, considering $X, A$ as a typical pair, and $M$ a linear subspace of $X$, we define $M^{\perp \perp}$ as the closure of $M$. This requires no topology in $X, A$, or $\Phi$, and resembles the Stone topology [1, p. 466] in this respect-and in fact admits a natural, joint generalization.

$M$ is closed if $M=M^{\perp \perp}$, and dense if $M^{\perp \perp}=X$.

\section{Proposition.}

3.31 The null-space of $T^{*}=(\text { range of } T)^{\perp}$

3.32 $T^{*}$ is single-valued only if and if the domain of $T$ is dense

$3.33 T^{*}$ is closed

$3.34 T^{* *}$ is the smallest closed linear relation containing $T$.

Here 3.31 is easily established on the definitions, and 3.32 follows from it by considering the null space of $T^{*-1}$. 3.33 is obvious, because any $M^{\perp}$. is closed, while 3.34 follows from 3.33 .

Turning to the adjoint of a sum, let $S$ and $T$ be two linear subspaces of $X \oplus Y$. It is quite elementary that

3.4

$$
S^{*}+T^{*} \subset(S+T)^{*} \text {. }
$$

The following gives an unsymmetric condition which insures the equality.

3.41 THEOREM. If the domain of $S^{*}=B$, and the domain of $S$ includes that of $T$, then

$$
(S+T)=S^{*}+T^{*}
$$

Proof. Let $(b, a) \in(S+T)^{*}$. Then there is an element $a_{1}$ such that $\left(b, a_{1}\right) \in S^{*}$. Let us show that $\left(b, a-a_{1}\right) \in T^{*}$. To this end, suppose $(x, t) \in T$. Then $(x, s) \in S$ for $s=S(x)$, and $(x, s+t) \in S+T$. Now

$$
\begin{aligned}
\left\langle x, a-a_{1}\right\rangle-\langle t, b\rangle & =\langle x, a\rangle-\left\langle x, a_{1}\right\rangle-\langle t, b\rangle \\
& =\langle x, a\rangle-\langle s, b\rangle-\langle t, b\rangle=\langle x, a\rangle-\langle s+t, b\rangle=0 .
\end{aligned}
$$

Thus $\left(b, a-a_{1}\right) \in T^{*}$, which, with $\left(b, a_{1}\right) \in S^{*}$ gives $(b, a) \in S^{*}+T^{*}$ as was to be shown.

Although our $T$ is not a function, we may adapt a symbolism usually used in a functional context, and write 


$$
X-X, \text { or } Y_{T}-X,
$$

to convey that $T$ is a linear subspace of $X \oplus Y$.

If we introduce $S$

$$
Y-{ }_{s} Z
$$

where $Z, C$ is another $(\Phi,-)$ dual pair, then

$$
S-{ }_{S T} Z \text {, and } C-{ }_{(S T) *} A \text {. }
$$

Since $A_{T^{*}}-B_{S^{*}}-C$ we also have $C-{ }_{T^{*} S^{*}} A$ and there arises the question of the relation of $(S T)^{*}$ and $T^{*} S^{*}$. In fact, it is quite elementary that $(S T)^{*} \supset T^{*} S^{*}$, but we wish to examine also the reverse inclusion, which is initiated by the following lemma. Here $f_{a}$ (for example) is the linear functional on $X$ defined by $f_{a}(x)=\langle x, a\rangle$, etc.

3.5 Lemma. Let $c \in C, a \in A$. Consider these linear functionals defined in $Y$

\subsection{1}

$$
f_{c} \circ S, f_{a} \circ T^{-1} \text {. }
$$

Then $(c, a) \in(S \circ T)^{*}$ if and only if these functionals are single-valued and agree on the intersection of their domains; and $(c, a) \in T^{*} \circ S^{*}$ if and only if they have a common extension to some $f_{b}, b \in B$.

Proof. The second assertion is the easier to show. If $(c, a) T^{*} \circ S^{*}$ then $(c, b) \in S^{*},(b, a) \in T^{*}$ for some $b \in B$. Let $y \in D(S) \cap D\left(T^{-1}\right)$ (' $D$ ' means 'domain'). I say these functionals (3.51) agree with $f_{b}$ for such $y$. Indeed, if $(y, z) \in S$ and $(y, x) \in T^{-1}$ then $f_{c}(z)=\langle z, c\rangle=\langle y, b\rangle=$ $\langle x, a\rangle=f_{a}(x)$.

Conversely, if $b$ having this property exists, then $(c, b) \in S^{*}$ and $(b, a) \in T^{*}$ or $(c, a) \in T^{*} \circ S^{*}$.

Now let $(c, a) \in(S \circ T)^{*}$, and let $y \in D(S) \cap D\left(T^{-1}\right)$. Let $(y, z) \in S$, $(x, y) \in T$. Then $(x, z) \in S \circ T$ and $\langle x, a\rangle=\langle z, c\rangle$, and these are generic elements of $\left(f_{a} \circ T^{-1}\right)(y),\left(f_{c} \circ S^{-1}\right)(y)$ respectively. Thus 3.51 are singlevalued, and agree on $D(S) \cap D\left(T^{-1}\right)$. The converse is obvious.

This establishes 3.5.

From this, a useful conclusion may be drawn.

3.52 Proposition. Suppose either that the domain of $S^{*}$ is $C$, or that the range of $T^{*}$ is $A$. Then

$$
(S \circ T)^{*}=T^{*} \circ S^{*} \text {. }
$$

Proof. Let $(c, a) \in(S \circ T)^{*}$. Consider the case in which the domain of $S^{*}$ is $c$. Then $(c, b) \in S^{*}$ for some $b$. Let $(y, z) \in S$. Then $\left(f_{c} \circ S\right)(y)=$ $\langle z, c\rangle=\langle y, b\rangle$, i.e., $f_{b}$ is an extension of $f_{c} \circ S$. Hence it is also an ex- 
tension of $f_{a} \circ T^{-1}$ (the latter confined, if need be, to the domain of $S+T^{-1}$.) We apply 3.5, and obtain $(c, a) \in T^{*} \circ S^{*}$.

If the range of $T^{*}$ is $A$, the proof is similar. But it may be reduced to the case treated, by using 3.22 , and the general fact $(U \circ V)^{-1}=V^{-1} \circ U^{-1}$.

We may now drop the ' $\circ$ ' again, which was reintroduced to make 3.5 easier to present.

3.6 Proposition. Let $U$ be a linear subspace of $X \oplus Y$, and $V$, of $Y \oplus Z$. If either the domain of $U^{* *}$ is $X$, or the range of $V^{* *}$ is $Z$, then $(V U)^{* *} \subset V^{* *} U^{* *}$.

Proof. In any case $U^{*} V^{*} \subset(V U)^{*}$ and $(V U)^{* *} \subset\left(U^{*} V^{*}\right)^{*}$. We think of $U^{*}$ as $S$ and $V^{*}$ as $T$ and apply 3.52, mutatis mutandis.

We recall (3.34) that $T$ is closed precisely when $T \supset T^{* *}$. The merit of our "many-valued" approach is that this criterion is available whether $T^{*}$ is single-valued or not.

3.7 THEOREM. Let $S$ and $T$ be linear relations as above. Suppose they are closed, and that either the domain of $T$ is $X$ or the range of $S$ is $Z$. Then $S T$ is closed.

Proof. By 3.6, we obtian $(S T)^{* *} \subset S^{* *} T^{* *}=S T$ provided the domain of $T$ is $X$ or the range of $S$ is $Z$, which suffices.

The relevance of the existence of resolvent values, to the question of closedness of polynomials in a (closed) operator, was noticed by Taylor [3] (see also [2, p. 56]).

3.8 THEOREM. Let $T$ be a closed linear subspace of $X \oplus X$, for which there is at least one $\lambda \in \Phi$ such that $T-\lambda$ has range $X$. Then $p(T)$, for any polynomial $p$ over $\Phi$, is closed.

Proof. By the algebraic Theorem 2.3 we have

$$
[p-p(\lambda)](T)=(T-\lambda) q(T)
$$

where $q$ is a polynomial of degree less than that of $p$. By 3.7 and an obvious inductive approach, we see that $[p-p(\lambda)](T)$ is closed. Now $[p-p(\lambda)](T)=p(T)-p(\lambda)$ by 2.35 , so the latter is closed. Note that $p(T)=U+V$ where $U=p(T)-p(\lambda), V=p(\lambda)$.

Now $(U+V)^{*} \supset U^{*}+V^{*}$ and so $(U+V)^{* *} \subset\left(U^{*}+V^{*}\right)^{*}$. Let $V^{*}$ be the $S$ of 3.41. Then its domain is the whole space, while $S^{*}=V$ and its domain is also the whole space. Thus $(U+V)^{* *} \subset U^{* *}+V^{* *}=$ $U+V$, so that $p(T)$ is closed. Of course, we also know that

$$
p(T)=p(\lambda)+(T-\lambda) p(T)
$$


which does not emerge from the proof given in [2].

4. Self-duality. When $X, A$ is a $(\Phi,-)$ dual pair and $A=X$, we speak of a self-dual pair. This situation presents two definitions of $M^{\perp}$, that given by 3.23 , and another, which we might call $\perp^{\perp} M$, given by 3.24 . These coincide if and only if

$$
\langle x, y\rangle=0 \text { if and only if }\langle y, x\rangle=0
$$

which, in turn, is equivalent to

4.11 There exists a $p \in \Phi$ such that $p \bar{p}=1$ and

$$
\langle y, x\rangle=p \overline{\langle x, y}\rangle \text { for all } x, y \in X \text {. }
$$

(We leave the proof of this equivalence to the reader. One should note that 4.1 for $X$ is transmitted, via 4.11, to $X \oplus X$, so that when $T \subset X \oplus X, T^{\perp}={ }^{\perp} T$ when 4.1 holds.)

The situation $M^{\perp} \neq{ }^{\perp} M$ would not be awkward if one had ${ }^{\perp}\left(M^{\perp}\right)=$ $\left({ }^{\perp} M\right)^{\perp}$, but for all we know this condition might be equivalent to 4.1. In any case, it does not hold in general (see 5.41).

We therefore assume 4.1 in this section.

Let $T$ be a linear subspace of $X \oplus X$. Then $W=T \mp T^{\perp}$ (see 2.6) is of interest, because for closed relations in Hilbert space, $W=X \oplus X$.

In general, the following relations hold:

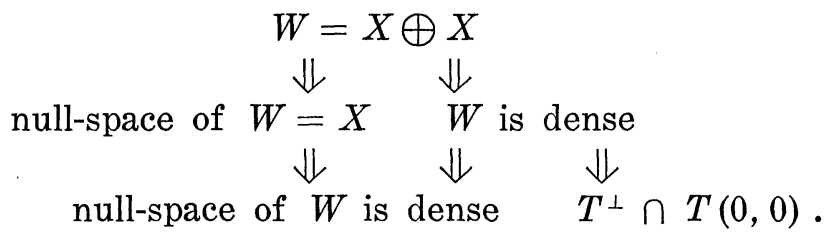

We proceed to generalize a proposition of von Neumann's [5].

4.3 Theorem. Let $T$ be closed. Let $W=T \mp T^{\perp}$ and suppose that the null-space of $W$ is all of $X$. Then the null-space of $1+T^{*} T$ is (0), the range is $X$, and $\left(T^{*} T\right)^{*}=T^{*} T$ (i.e., $T^{*} T$ is self-adjoint.)

Proof. Let $U$ (in 2.61) $=T$, and $V=T^{\perp}$. Then $-V^{-1}=T^{*}$. Therefore the range of $1+T^{*} T$ is the null-space of $W$, that is, $X$. Moreover, the null-space of $\left(1+T^{*} T\right)^{*}$ is (by 3.31) (range of $\left.1+T^{*} T\right)^{\perp}$, which is $(0)$.

We know that $T^{*} S^{*} \subset(S T)^{*}$ in general, so if we set $S=T^{*}$, $S^{*}=T^{* *}=T$, we get $T^{*} T \subset\left(T^{*} T\right)^{*}$, or $1+T^{*} T \subset\left(1+T^{*} T\right)^{*}$. Here we have used 3.41 .

Considering 2.02, and what we know about the null-spaces and ranges, we conclude that $1+T^{*} T=\left(1+T^{*} T\right)^{*}, T^{*} T=\left(T^{*} T\right)^{*}$.

We have already defined $T$ to be self-adjoint if $T=T^{*}$. We call 
$T$ unitary if $T^{*}=T^{-1}$. We say nothing about single-valuedness. In the Hilbert-space-situation, there are no unitary linear relations except those single-valued relations which are usually called unitary, as the following shows.

4.4 Proposition. $T^{-1} \subset T^{*}$ if and only if $\langle x, x\rangle=\langle y, y\rangle$ for all $(x, y) \in T$. If $T^{*}=T^{-1}$ and $T \mp T^{\perp}=X \oplus X$ then the domain and range of $T$ both equal $X$.

Proof. The statement about $\langle x, x\rangle$ and $\langle y, y\rangle$ is obviously true.

Now assume $T \mp T^{\perp}=X \oplus X$ and $T^{*}=T^{-1}$. Let $y \in X$. Then $(0, y)=(x, t)+(-x, y-t)$ where $(x, t) \in T$ and $(-x, y-t) \in T^{\perp}=$ $\left(-T^{*}\right)^{-1}=-T$, or $(x, y-t) \in T$. Then $(2 x, y) \in T$, or the given $y$ is in the range of $T$. Now the things assumed about $T$ are inherited by $T^{-1}$ so that the range of $T^{-1}$ is also $X$.

Returning briefly to the Hilbert-space-situation, if $T^{*}=T^{-1}$ then $T$ is closed and so $T \mp T^{\perp}$ does equal $X \oplus X$, whence $T$ is unitary in the usual sense.

To generalize the formal aspects of the Cayley transform [4] we assume now that $\Phi$ contains an element $i$ such that $i^{2}=-1$ and $\bar{i}=-i$.

Cayley's map sends $X \oplus X$ onto $X \oplus X$ thus

$$
C(x, y)=(x-i y, x+i y) .
$$

Its third iterate is scalar, and it preserves orthogonality, etc. If $T \subset X \oplus X$ then

$$
C(T)=\{(s-i t, s+i t):(s, t) \in T\}
$$

is the Cayley transform of $T$.

We list several elementary properties.

$$
\begin{aligned}
& S \subset T \Leftrightarrow C(S) \subset C(T) \\
& C(-T)=C(T)^{-1} \\
& C\left(T^{-1}\right)=-C(T)^{-1} \\
& C\left(T^{\perp}\right)=C(T)^{\perp} \\
& C\left(T^{*}\right)=C(T)^{*-1} .
\end{aligned}
$$$$
4.55
$$

4.6 Theorem. $T \subset T^{*}$ if and only if $C(T)^{-1} \subset C(T)^{*}, T=T^{*}$ if and only if $C(T)$ is unitary.

If $C^{2}(T)$ were unitary, and we were in Hilbert space, then $T$ would have a spectral resolution, but $C^{2}(T)$ is unitary if and only if $T^{*}=-T$.

The spectral mapping theorem holds for this Cayley transform:

$$
\sigma(C(T))=\left\{(1+i \tau)(1-i \tau)^{-1}: \tau \in(T)\right\}
$$


with the following understanding: $\infty \in \sigma(S)$ means $0 \in \sigma\left(S^{-1}\right), 2 / 0=\infty$, $(1+i \infty)(1-i \infty)^{-1}=-1$. Moreover, eigenvalues correspond to eigenvalues.

The set consisting of the spectrum of $T$, plus the symbol $\infty$ if $0 \in \sigma\left(T^{-1}\right)$ we call, following Taylor, the augumented spectrum. The augmented spectrum thus contains $\infty$ whenever $T$ is not single-valued.

5. Hilbert space. In Hilbert space $X,(\Phi=$ complex numbers), selfadjoint linear relations $T$ may be analyzed in just the same way as the single-valued ones are, by von Neumann, in [4]. The general theory is perfect in a way that the usual theory is not: every unitary operator is the Cayley transform of a unique self-adjoint linear relation, and conversely (4.6).

However, rather than repeat the application of the Cayley transform method, we prefer to analyze the general self-adjoint linear relation in term of self-adjoint operators.

If $T$ is a closed linear subspace of $X \oplus X, X$ being a Hilbert space (as shall be assumed in all of this section) then

$$
T=T_{\infty} \pm T_{1}
$$

where $T_{\infty}, T_{1}$ are orthogonal closed linear subspaces (so we write ' \pm ' instead of ' $\mp$ ') and $T_{\infty}=T \cap(\{0\} \oplus X)$. Thus $T_{\infty}$ has only 0 in its domain, while its range is $T(0)$ (see $\S 2) . T(0)$ is closed, since $T_{\infty}=$ $\{0\} \oplus T(0)$. The domain of $T_{1}$ is the domain of $T$, and $T_{1}$ is singlevalued.

5.2 Lemma. $T(0)=\left(\operatorname{dom} T^{*}\right)^{\perp}$, dom $T_{1}$ is dense in $T^{*}(0)^{\perp}$, and the range of $T_{1}$ lies in $T(0)^{\perp}$.

Proof. 3.31 tells us that $T^{*^{-1}}(0)=\left(\operatorname{dom} T^{-1}\right)^{\perp}$. We can replace $T$ here by $T^{-1}$, and then replace $T^{*}$ by $T$ since $T$ is closed. Thus $T(0)=$ $\left(\operatorname{dom} T^{*}\right)^{\perp}$. From $T^{*}(0)=(\operatorname{dom} T)^{\perp}$ we obtain $(\operatorname{dom} T)^{-1}=T^{*}(0)^{\perp}$, and thus the second assertion. Finally, if $(x, y) \in T_{1}$, and $(0, z) \in T_{\infty}$ then $(x, y) \perp(0, z)$, because $T_{1}$ is the orthogonal complement of $T_{\infty}$ relative to $T$. Hence $\langle y, z\rangle=0$.

5.3 Theorem. Let $T$ be a self-adjoint linear subspace of $X \oplus X$. Let $T=T_{\infty} \pm T_{1}$ as above. Then

$$
X=Y \pm Z
$$

and $T_{\infty}$ consists of all pairs $(0, y), y \in Y$ while $T_{1}$ is a closed linear operator whose domain is dense in $Z$, and whose range is in $Z$. $T_{1}$, restricted to $Z$, coincides with a self-adjoint linear operator in $Z$. 
Proof. Let $Y=T(0), Z=T(0)^{\perp}$. Then the domain of $T_{1}$ is dense in $T^{*}(0)^{\perp}=Y^{\perp}=Z$ and the range lines in $T(0)^{\perp}=Z$, all by 5.2 .

Suppose that $(z, w) \in S^{*}$ where $S$ is $T_{1}$ restricted to $Z$. Then $\langle x, w\rangle=\langle v, z\rangle$ for all $(x, v) \in T_{1}$. Each $(x, u) \in T$ is of the form $(x, y+v)$ where $y \in T(0)$ and $(x, v) \in T_{1}$. Now $\langle y, z\rangle=0$, so $\langle x, w\rangle=$ $\langle y+v, z\rangle$ for all $(x, y+v) \in T$. It follows that $(z, w) \in T^{*}=T$. But since $z, w \in Z$ we have $(z, w) \in T_{1}$. This proves 5.3.

We return here to the question raised in second paragraph of $\S 4$, because a counterexample in a Hilbert space context is more desireable than any other. Let $X=L_{2}[0,2]$, in which the inner product will be denoted by $\langle$,$\rangle , and orthogonality, by \perp$. Select a bounded operator $T$, domain $X$, range dense, with single-valued inverse, and define a selfdual pairing by means of the formula

$$
[f, g]=\langle T f, g\rangle=\left\langle f, T^{*} g\right\rangle .
$$

The associated orthogonality will be denoted by ' $\circ$ ' to prevent confusion with ' $\perp$ ' already present.

5.41 Proposition. It is possible to select $T$ and $M$ (a linear subspace of $X$ ) such that

$$
{ }^{\circ}\left(M^{\circ}\right)=M \text { but }\left({ }^{\circ} M\right)^{\circ} \neq M \text {. }
$$

Before deciding on a specific $T$ we shall establish

5.43 Lemma. ${ }^{\circ}\left(M^{\circ}\right)$ is the closure of $M$ in the norm $\|x\|_{T}=\|T x\|$ $[4,298]$, and $\left({ }^{\circ} M\right)^{\circ}$ is the closure of $M$ in $\|\cdot \cdot\|_{T^{*}}$.

Proof. $M^{\circ}=\{a:[M, a]=0\}={ }^{\perp}(T M)$, and ${ }^{\circ} M={ }^{\perp}\left(T^{*} M\right)$. Consequently ${ }^{\circ}\left(M^{\circ}\right)={ }^{\perp}\left[T^{* \perp}(T M)\right]$, and so $g \in{ }^{\circ}\left(M^{\circ}\right)$ precisely when $g \perp T^{* \perp}(T M)$ or $T g \perp{ }^{\perp}(T M)$, i.e.,

$$
T g \in(T M)^{\perp \perp}=\overline{T M} .
$$

But this characterizes the closure of $M$ in $\|\cdots\|_{T}$, and this observation suffices to establish 5.43 .

Now we select $T=J$ where

$$
(J f)(t)=\int_{0}^{t} f(\tau) d \tau .
$$

This $J$ meets our requirement for $T$. We have

$$
\left(J^{*} f\right)(t)=\int_{t}^{2} f(\tau) d \tau,
$$

whence $J^{*}=E-J$ where $E$ is the projection on the constant functions 
in $X$.

Let $N$ be the linear subspace of those functions that vanish on $[1,2]$. Let

$$
h(t)= \begin{cases}1 & 0 \leqq t<1 \\ 0 & 0 \leqq t \leqq 2\end{cases}
$$

Then $h \in N$ and $M=N \cap\{h\}^{\perp} \neq N$. Thus $E M=(0)$. It is easy to establish, in the order given, the following: $J M \subset N, J^{*} N \subset N, \overline{J M}=N$, $\overline{J^{*} M}=N$.

Then one observes that $J f \in N$ implies $f \in M$ while $J^{*} f \in N$ implies $f \in N$, (and each converse holds, because $J M \subset N, J^{*} N \subset N$.) Using 5.44 as a criterion for $J g \in{ }^{\circ}\left(M^{\circ}\right)$ we obtain ${ }^{\circ}\left(M^{\circ}\right)=M,\left({ }^{\circ} M\right)^{\circ}=N$.

\section{BIBLIOGRAPHY}

1. Richard Arens, A generalization of normed rings, Pacific J. Math., 2 (1952), 455-471.

2. E. Hille and R.S. Phillips, Functional Analysis and Semi-groups, A.M.S. Collow. Publ., 31.

3. A. E. Taylor, Spectral Analysis of closed distributive operators, Acta Math., 84 (1950), 189-224.

4. J. von Neumann, Über adjungierte Funktional-operatoren, Ann. Math., 33, (1932), 294310 .

UNiversity of CALIForNia, Los ANGELES 



\section{PACIFIC JOURNAL OF MATHEMATICS}

\section{EDITORS}

Ralph S. Phillips

Stanford University

Stanford, California

F. H. BrownelL

University of Washington

Seattle 5 , Washington
A. L. Whiteman

University of Southern California Los Angeles 7. California

L. J. PAIGE

University of California

Los Angeles 24, California

ASSOCIATE EDITORS
E. F. BECKENBACH
D. DERRY
H. L. ROYDEN
E. G. STRAUS
T. M. CHERRY
M. OHTSUKA
E. SPANIER
F. WOLF

\section{SUPPORTING INSTITUTIONS}

UNIVERSITY OF BRITISH COLUMBIA

CALIFORNIA INSTITUTE OF TECHNOLOGY

UNIVERSITY OF CALIFORNIA

MONTANA STATE UNIVERSITY

UNIVERSITY OF NEVADA

NEW MEXICO STATE UNIVERSITY

OREGON STATE COLLEGE

UNIVERSITY OF OREGON

OSAKA UNIVERSITY

UNIVERSITY OF SOUTHERN CALIFORNIA
STANFORD UNIVERSITY

UNIVERSITY OF TOKYO

UNIVERSITY OF UTAH

WASHINGTON STATE COLLEGE,

UNIVERSITY OF WASHINGTON

AMERICAN MATHEMATICAL SOCIETY

CALIFORNIA RESEARCH CORPORATION

HUGHES AIRCRAFT COMPANY

SPACE TECHNOLOGY LABORATORIES

NAVAL ORDNANCE TEST STATION

Printed in Japan by International Academic Printing Co., Ltd., Tokyo, Japan

Reprinted 1966 in the United States of America 


\section{Pacific Journal of Mathematics}

\section{Vol. 11, No. $1 \quad$ November, 1961}

A. A. Albert, Generalized twisted fields ............................ 1

Richard Arens, Operational calculus of linear relations ................... 9

John Herbert Barrett, Disconjugacy of a self-adjoint differential equation of the fourth order ....................................... 25

Paul Richard Beesack, Hardy's inequality and its extensions ............... 39

Julius Rubin Blum and David Lee Hanson, On invariant probability measures.

II .............................................

Robert Allen Bonic, Symmetry in group algebras of discrete groups.......... 73

R. Creighton Buck, Multiplication operators ...................... 95

Jack Gary Ceder, Some generalizations of metric spaces ................. 105

Meyer Dwass, Random crossings of cumulative distribution functions ......... 127

Albert Edrei, Wolfgang H. J. Fuchs and Simon Hellerstein, Radial distribution and

deficiencies of the values of a meromorphic function ............... 135

William Cassidy Fox, Harmonic functions with arbitrary local singularities ..... 153

Theodore Thomas Frankel, Manifolds with positive curvature ............... 165

Avner Friedman, A strong maximum principle for weakly subparabolic

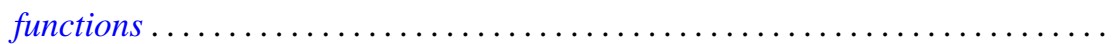

Watson Bryan Fulks and J. O. Sather, Asymptotics. II. Laplace's method for

multiple integrals ......................................

Adriano Mario Garsia and Eugene Richard Rodemich, An embedding of Riemann

surfaces of genus one ..................................... 193

Irving Leonard Glicksberg, Weak compactness and separate continuity......... 205

Branko Grünbaum, On a conjecture of H. Hadwiger .................. 215

Frank J. Hahn, On the action of a locally compact group on $E_{n} \ldots \ldots \ldots \ldots \ldots . . \ldots 221$

Magnus R. Hestenes, Relative hermitian matrices ..................... 225

G. K. Kalisch, On similarity invariants of certain operators in $L_{p} \ldots \ldots \ldots \ldots .247$

Yitzhak Katznelson and Walter Rudin, The Stone-Weierstrass property in Banach

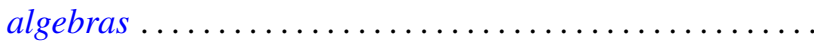

Samir A. Khabbaz, The subgroups of a divisible group $G$ which can be represented as intersections of divisible subgroups of $G \ldots \ldots \ldots \ldots \ldots \ldots \ldots \ldots \ldots . \ldots \ldots 7$

Marvin Isadore Knopp, Construction of a class of modular functions and forms .......................................... 275

Charles Alan McCarthy, Commuting Boolean algebras of projections .......... 295

T. M. MacRobert, Transformations of series of E-functions ................ 309

Heinz Renggli, An inequality for logarithmic capacities ................. 313

M. S. Robertson, Applications of the subordination principle to univalent functions .......................................... 315

David Sachs, Partition and modulated lattices ..................... 325

Frank S. Scalora, Abstract martingale convergence theorems ............... 347

Elbert A. Walker, Torsion endomorphic images of mixed Abelian groups ........ 375

Morgan Ward, The prime divisors of Fibonacci numbers................. 379

Charles R. B. Wright, On the nilpotency class of a group of exponent four....... 387 